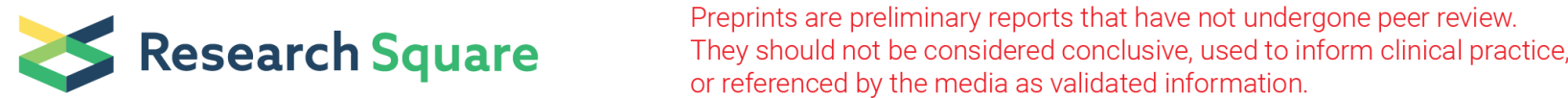

\section{Development and characterization of 57 SNP markers in Misgurnus anguillicaudatus}

Guiyun Huang

Guangdong Ocean University

Fengying Gao

Chinese Academy of Fishery Sciences Pearl River Fisheries Research Institute

Zhigang Liu

Chinese Academy of Fishery Sciences Pearl River Fisheries Research Institute

Jianmeng Cao

Chinese Academy of Fishery Sciences Pearl River Fisheries Research Institute

\section{Gang Chen}

Guangdong Ocean University

Maixin Lu ( $\sim$ mx-lu@163.com)

Chinese Academy of Fishery Sciences Pearl River Fisheries Research Institute

\section{Research Article}

Keywords: Misgurnus anguillicaudatus, 2b-RAD sequencing, Single nucleotide polymorphism (SNP), SNaPshot

Posted Date: February 18th, 2021

DOI: https://doi.org/10.21203/rs.3.rs-201840/v1

License: (c) (1) This work is licensed under a Creative Commons Attribution 4.0 International License. Read Full License 


\section{Abstract}

The dojo loach Misgurnus anguillicaudatus is an endemic freshwater species to Asia. The effective conservation and molecular-aided selection of $M$. anguillicaudatus have been limited without sufficient molecular markers. In this study, 112 novel single nucleotide polymorphisms (SNPs) were screened based on 2b-RAD sequencing database, and 57 SNP markers were developed and characterized by genotyping 40 individuals using SNaPshot method. The observed heterozygosity $(\mathrm{Ho})$ ranged from 0.025 to 0.675 , while the expected heterozygosity $(\mathrm{He})$ varied from 0.025 to 0.500 . The minor allele frequency $(M A F)$ ranged from 0.013 to 0.500 . Among these SNPs, 18 loci were found to deviate significantly from the Hardy-Weinberg equilibrium after Bonferroni correction $(P<0.05)$. The first set of SNP markers developed from $M$. anguillicaudatus will provide valuable information in further population genetic analysis and natural resource conservation.

\section{Background}

The dojo loach Misgurnus anguillicaudatus, a member of the family Cobitidae, is an endemic freshwater species in Asia and is widely distributed along the eastern coasts of the Asian continent (Li et al. 2008; Zeng et al. 2012). For delicious meat and traditional Chinese medicine values, the doji loach has become one of the most important economic fish in several East Asian countries. In addition to their edible value, loach is regarded as an excellent animal model to explain the biological origin and evolutionary significance of polyploidization because of its complex ploidy composition (Zhou et al. 2014; Zhong et al. 2019). Despite its economic importance, dojo loaches for human consumption are mostly obtained through the exploitation of wild populations. In recent years natural populations have declined dramatically because of over-exploitation and habitat destruction. Therefore, it is urgent to perform population genetic investigation on $M$. anguillicaudatus to protect the natural resources.

The research of genetic markers in M. anguillicaudatus has mainly concentrated on mitochondrial DNA and microsatellite marker at present (Zhang et al. 2013; Fujimoto et al. 2017; Lee et al. 2018). Owing to their wide genomic distribution and high level of polymorphism, single nucleotide polymorphisms (SNPs) have gradually become one of the most important DNA markers for genetic studies (Vignal et al. 2002; Seeb et al. 2011). Unfortunately, limited reports are available for M. anguillicaudatus on SNPs. To better understand the genetic background and enrich the molecular markers, we firstly developed a set of SNP markers by SNaPshot technique in M. anguillicaudatus.

A total of 36,916 putative SNPs were identified using 2b-RAD genotyping method, and 112 novel SNP markers were randomly selected and evaluated to test polymorphic. Forty caudal fin samples of diploid M. anguillicaudatus were randomly collected from Qingyuan City, Guangdong Province, China. Genomic DNA was extracted from tissue samples using the HiPure Mollusc DNA Mini Kit (Magen, China) according to the manufacturer's instruction. Each PCR reaction (10

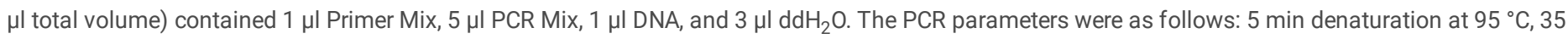
cycles of $20 \mathrm{~s}$ at $94{ }^{\circ} \mathrm{C}, 30 \mathrm{~s}$ at $60^{\circ} \mathrm{C}$ and $30 \mathrm{~s}$ at $72{ }^{\circ} \mathrm{C}$, and an extension step of $3 \mathrm{~min}$ at $72{ }^{\circ} \mathrm{C}$. Next, the purification and extension of the PCR products were performed using the SNaPshot technique of ABI Company. Finally, genotyping was conducted using ABI 3730XL DNA Analyzer (Applied Biosystems). For validated loci, statistics including MAF, $H_{O}$ and $H e$ were calculated in GenAlex v6.5. The polymorphism information content (PIC) and deviations from the Hardy-Weinberg equilibrium (HWE) were estimated using GenePop 3.2.

Of the 112 potential SNP markers, 57 loci were found to be polymorphic and bi-allelic. The Ho and Hevaried from 0.025 to 0.675 and 0.025 to 0.500 , respectively. The MAF ranged from 0.013 to 0.500 . The PIC ranged from 0.024 to 0.375 (Table1). Among these SNPs, 18 loci showed significant deviations from the Hardy-Weinberg equilibrium after Bonferroni correction $(P<0.05)$. To our knowledge, this is the first development of SNP markers in $M$. anguillicaudatus, and these novel markers offer a valuable tool for future studies of population conservation and natural resource conservation in this species.

\section{Declarations}

\section{Conflicts of interest}

The authors declare no competing financial interests.

\section{Funding information}

This research was funded by China Agriculture Research System (CARS-46) and the National Key R\&D Program of China (No. 2018YFD0901201). We thank Yaosen Qian who provided loach tissue samples for this research.

\section{Authors' contribution}

Conceived and designed the experiments: GC ML. Performed the experiments, analyzed the genetic data: GH. Contributed reagents/materials/analysis tools: FG ZL JC. Wrote the paper: GH ML. All authors read, revised, and approved the manuscript.

\section{Data Availability}

The data used to support the findings of this study are available from the article and its supplementary information.

\section{Ethics approval(Ethics)}

All experimental procedures for loach handling were approved by the Animal Care and Ethics Committee of the Pearl River Fisheries Research Institute, Chinese Academy of Fishery Sciences. 
Consent to Participate (Ethics)

This study was conducted at Pearl River Fisheries Research Institute, Chinese Academy of Fishery Sciences. The institution and all authors agreed to participate in this research.

\section{Consent to Publish (Ethics)}

The Author confirms:

- that the work described has not been published before;

- that it is not under consideration for publication elsewhere;

- that its publication has been approved by all co-authors if any;

- that its publication has been approved explicitly by the responsible authorities at the institution where the work is carried out.

The authors agree to publication in the journal Conservation Genetics Resources.

\section{References}

Fujimoto T, Yamada A, Kodo Y, Nakaya K, Okubo-Murata M, Saito T, Ninomiya K, Inaba M, Kuroda M, Arai K, Murakami M (2017) Development of nuclear DNA markers to characterize genetically diverse groups of Misgurnus anguillicaudatus and its closely related species. Fish Sci 83: 743-756.

Lee SY, Bang IC, Nam YK (2018) Complete mitochondrial genome of albino cyprinid loach, Misgurnus anguillicaudatus (Cypriniformes: Cobitidae). Conserv Genet Resour 10: 507-510.

Li YJ, Yin J, Wang JB, Yuan X, Wei J, Sun XW, Arai K (2008) A study on the distribution of polyploid loaches in China. Nippon Suisan Gakkaishi 74:177-182.

Seeb JE, Carvalho G, Hauser L, Naish K, Roberts S, Seeb LW (2011) Single-nucleotide polymorphism (SNP) discovery and applications of SNP genotyping in nonmodel organisms. Mol Ecol Resour 11: 1-8.

Vignal A, Milan D, SanCristobal M, Eggen A (2002) A review on SNP and other types of molecular markers and their use in animal genetics. Genet Sel Evol 34: 275-305.

Zeng LG, Wang JH, Sheng JQ, Gu Q, Hong YJ (2012) Molecular characteristics of mitochondrial DNA and phylogenetic analysis of the loach (Misgurnus anguillicaudatus) from the Poyang Lake. Mitochondrial DNA 23: 187-200.

Zhang XJ, Wang WM, Huang SQ, Chen GM, Bai XH, Cao XJ (2014) The complete mitochondrial genomes of natural diploid and tetraploid loaches Misgurnus anguillicaudatus (Cypriniformes: Cobitidae). Mitochondrial DNA 25: 196-197.

Zhong J, Yi S, Ma L, Wang W (2019) Evolution and phylogeography analysis of diploid and polyploid Misgurnus anguillicaudatus populations across. China Proc Biol Sci 286: 20190076.

Zhou X, Yu Y, Li Y, Wu J, Zhang X, Guo X, Wang W (2014) Comparative analysis of mitochondrial genomes in distinct nuclear ploidy loach Misgurnus anguillicaudatus and its implications for polyploidy evolution. PLoS One 9: e92033.

\section{Tables}

Table 1 Characteristics of SNP markers in Misgurnus anguillicaudatus 


\begin{tabular}{|c|c|c|c|c|c|c|c|c|c|}
\hline $\begin{array}{l}\text { Prime } \\
\text { ID }\end{array}$ & Primer sequence $\left(5^{\prime}-3^{\prime}\right)$ & $\begin{array}{l}\text { Size } \\
\text { (bp) }\end{array}$ & $\begin{array}{l}\text { SNP } \\
\text { type }\end{array}$ & $\mathrm{Ne}$ & Ho & $\mathrm{He}$ & $P I C$ & MAF & PHИ \\
\hline SNP4 & $\begin{array}{l}\text { F: ACTCTTTGAGGTTGACCCAG } \\
\text { R: TTTGCGGGATGCTACATCTG } \\
\text { E: GAAGGAGCCACGGTTAAATC }\end{array}$ & 280 & $\mathrm{G} / \mathrm{A}$ & 1.220 & 0.200 & 0.180 & 0.164 & 0.100 & 1.00 \\
\hline SNP9 & $\begin{array}{l}\text { F: GGGATCATCTGAAAGACTCG } \\
\text { R: CATAACTGCCCTATCATCTG } \\
\text { E: gactAACCTACTGTTCCTCCCATA }\end{array}$ & 278 & $\mathrm{C} / \mathrm{A}$ & 1.140 & 0.079 & 0.123 & 0.116 & 0.066 & 0.13 \\
\hline SNP10 & $\begin{array}{l}\text { F: GTGAATTACAGTGCTGACGG } \\
\text { R: TGTTGGCACGTTCAACAGTC } \\
\text { E: gactAACCTACTGTTCCTCCCATA }\end{array}$ & 279 & $\mathrm{G} / \mathrm{C}$ & 1.280 & 0.200 & 0.219 & 0.195 & 0.125 & 0.47 \\
\hline SNP14 & $\begin{array}{l}\text { F: CACAAGCATAGGACTCTGAC } \\
\text { R: AGGCAGTTTAGTAGGTGTTG } \\
\text { E: tgactgactgactATCCCAACTTTGTTACCGCCA }\end{array}$ & 292 & $\mathrm{C} / \mathrm{A}$ & 1.342 & 0.150 & 0.255 & 0.222 & 0.150 & 0.02 \\
\hline SNP15 & $\begin{array}{l}\text { F: GTGCTATGCTGTACTGTCTG } \\
\text { R: CATCTCTTCTGCACTAGGTC } \\
\text { E: actgactgactgactGGAAAGTTCAGCCTGAACCAG }\end{array}$ & 276 & $\mathrm{C} / \mathrm{A}$ & 1.105 & 0.100 & 0.095 & 0.090 & 0.050 & 1.00 \\
\hline SNP23 & $\begin{array}{l}\text { F: TGTCCCTTTAAGCTTCCCTG } \\
\text { R: CACTTTGTGTTTGACCCCTG } \\
\text { E: gactgactgactgactAAATATTCAAACATACCTCCATACGG }\end{array}$ & 298 & $\mathrm{G} / \mathrm{A}$ & 1.105 & 0.100 & 0.095 & 0.090 & 0.050 & 1.00 \\
\hline SNP29 & $\begin{array}{l}\text { F: GCATTGGATTGCATCTGGTG } \\
\text { R: GCTCTGTGGTTTATAAAGGG } \\
\text { E: actgactgactgactgactgactGATGGCCACAGAAACTCCAGG }\end{array}$ & 278 & $\mathrm{~A} / \mathrm{C}$ & 1.753 & 0.375 & 0.430 & 0.337 & 0.300 & 0.30 \\
\hline SNP30 & $\begin{array}{l}\text { F: CACAGTAAGGTTAGAGGGAG } \\
\text { R: TAGGTAAAGAAGCGCAGCAG } \\
\text { E: ctgactgactgactgactgactgactTGATGTACCCCACCTCCTCTTC }\end{array}$ & 280 & $\mathrm{C} / \mathrm{T}$ & 1.471 & 0.200 & 0.320 & 0.269 & 0.200 & 0.02 \\
\hline SNP37 & $\begin{array}{l}\text { F: TGCCATGTTCTTCCGGTTTG } \\
\text { R: CACCCGAAAGTGAACACATC } \\
\text { E: actgactgactgactgactgactgactgactgactCTGTAATGAGTGTGATCTCT }\end{array}$ & 284 & $\mathrm{G} / \mathrm{T}$ & 1.161 & 0.100 & 0.139 & 0.129 & 0.075 & 0.18 \\
\hline SNP42 & $\begin{array}{l}\text { F: TGGATAGAGTGTGTGTATCG } \\
\text { R: TCACCCATCAAGCCCAAATG } \\
\text { E: gactgactgactgactgactgactgactgactTGTGTGTATCGATAAATACTGTAACT }\end{array}$ & 279 & $\mathrm{G} / \mathrm{A}$ & 1.250 & 0.175 & 0.200 & 0.180 & 0.113 & 0.39 \\
\hline SNP48 & $\begin{array}{l}\text { F: ACTCTGCAATCTTGGCCATC } \\
\text { R: ATGACGACGATTGGGATGAC } \\
\text { E: ctgactgactgactgactgactgactgactgactgactAACCTGAACCGATTCTCCAGCTT }\end{array}$ & 282 & $\mathrm{G} / \mathrm{A}$ & 1.809 & 0.675 & 0.447 & 0.347 & 0.338 & 1.00 \\
\hline SNP50 & $\begin{array}{l}\text { F: TGTTTACTGTGGCGCTGATG } \\
\text { R: TTCACTTTGGGCTTTGGGAG } \\
\text { E: } \\
\text { gactgactgactgactgactgactgactgactgactgactgactGACCCCAACCCTCCCTCCGC }\end{array}$ & 280 & $\mathrm{C} / \mathrm{T}$ & 1.503 & 0.175 & 0.335 & 0.312 & 0.188 & 1.00 \\
\hline SNP60 & $\begin{array}{l}\text { F: ATAGCACCTTTCAGGAACCC } \\
\text { R: TTCTGGTACAGCACACAAGG } \\
\text { E: gactgactgactgactgactgactgactgactgactCCTGTACAGGACACACAATT }\end{array}$ & 279 & $\mathrm{C} / \mathrm{A}$ & 1.632 & 0.275 & 0.387 & 0.279 & 0.225 & 0.06 \\
\hline SNP65 & $\begin{array}{l}\text { F: GTGTGGGAGAAGAACGTATG } \\
\text { R: AGCGAAAGTGAGACAGAGAG } \\
\text { E: gactgactgactgactTGGATGAGAGAGTGCATGAGTCG }\end{array}$ & 279 & $\mathrm{C} / \mathrm{T}$ & 1.584 & 0.231 & 0.369 & 0.301 & 0.204 & 0.02 \\
\hline SNP71 & $\begin{array}{l}\text { F: ACTCCCTGGAGTTAAAGCTG } \\
\text { R: TGATCCCAGCACAATAGGAG } \\
\text { E: CACTGGAGGTGCTTGCAAAA }\end{array}$ & 278 & $\mathrm{C} / \mathrm{G}$ & 1.105 & 0.100 & 0.095 & 0.090 & 0.050 & 0.77 \\
\hline
\end{tabular}

Table 1 (continued) 


\begin{tabular}{|c|c|c|c|c|c|c|c|c|c|}
\hline $\begin{array}{l}\text { Prime } \\
\text { ID }\end{array}$ & Primer sequence( $\left.5^{\prime}-3^{\prime}\right)$ & $\begin{array}{l}\text { Size } \\
\text { (bp) }\end{array}$ & $\begin{array}{l}\text { SNP } \\
\text { type }\end{array}$ & $\mathrm{Ne}$ & Ho & $\mathrm{He}$ & $P I C$ & MAF & $P H$ \\
\hline SNP72 & $\begin{array}{l}\text { F: AGCACTTCCTTTGTGCTACG } \\
\text { R: CACTGTCATGTCTGATAGCC } \\
\text { E: gactAGGGATACAGGAGTGCGCGT }\end{array}$ & 280 & $\mathrm{G} / \mathrm{A}$ & 1.026 & 0.025 & 0.025 & 0.024 & 0.013 & $1 . C$ \\
\hline SNP73 & $\begin{array}{l}\text { F: TAATGGCACGGGAAGTGAAG } \\
\text { R: AGCCTTGCAGAGAGATTTGG } \\
\text { E: gactgactGAGAGATTTGGAGGATAGT }\end{array}$ & 280 & $\mathrm{G} / \mathrm{A}$ & 1.220 & 0.100 & 0.180 & 0.164 & 0.100 & $0 . C$ \\
\hline SNP74 & $\begin{array}{l}\text { F: TTCACCTGCTGCATGTCTTC } \\
\text { R: GTGAAACTCAGAGGAGACAG } \\
\text { E: actgactgactAGGAGAGAGAAGAGGAGATTG }\end{array}$ & 272 & $\mathrm{C} / \mathrm{T}$ & 1.980 & 0.400 & 0.495 & 0.372 & 0.450 & 0.1 \\
\hline SNP75 & $\begin{array}{l}\text { F: TTAGAAGCCCTGCACTTGTC } \\
\text { R: CTCGAAGAAGAGTCTACAGG } \\
\text { E: tgactgactgactgactTGATAAATCTTCAAAACTTCAAC }\end{array}$ & 275 & $A / G$ & 1.133 & 0.125 & 0.117 & 0.110 & 0.063 & 0.7 \\
\hline SNP76 & $\begin{array}{l}\text { F: CATTGTTCTGTAGGCAGACC } \\
\text { R: GCTGTTTGGCATGGTTTTTG } \\
\text { E: tgactgactgactgactgactATTTTAACGTACCCCAGGAAAAT }\end{array}$ & 280 & $T / G$ & 1.956 & 0.400 & 0.489 & 0.369 & 0.450 & 0.2 \\
\hline SNP77 & $\begin{array}{l}\text { F: TTGAGGCCCTTTCTATGGAC } \\
\text { R: TTTGCCGAGCTTATCCTGTG } \\
\text { E: gactgactgactgactgactgactgactTGTCTGAGGGAGATGCAGTG }\end{array}$ & 280 & $\mathrm{G} / \mathrm{T}$ & 1.809 & 0.275 & 0.447 & 0.347 & 0.338 & $0 . C$ \\
\hline SNP78 & $\begin{array}{l}\text { F: TGAATACACCGAACTCTGGG } \\
\text { R: CACTATCAAGCTGATCTCCG } \\
\text { E: ctgactgactgactgactgactgactgactCATAGGAGTCTGTGTCCGAGTT }\end{array}$ & 288 & $A / G$ & 1.025 & 0.025 & 0.025 & 0.024 & 0.013 & $1 . C$ \\
\hline SNP79 & $\begin{array}{l}\text { F: TCTGTGGTGTTGTTTTTCCC } \\
\text { R: TTGGTGGAAAAAGGACCAG } \\
\text { E: gactgactgactgactgactgactgactgactgactTAGGGGGAGCTCTCGTCTAC }\end{array}$ & 281 & $\mathrm{C} / \mathrm{T}$ & 1.105 & 0.050 & 0.095 & 0.090 & 0.050 & $0 . C$ \\
\hline SNP80 & $\begin{array}{l}\text { F: TGACATCAGCAACCACAGAC } \\
\text { R: TTTGCCGTATGGGACAGTTG } \\
\text { E: actgactgactgactgactgactgactgactgactgactTTTGGTCTGGAGAAGGAAGCG }\end{array}$ & 280 & $\mathrm{G} / \mathrm{T}$ & 1.051 & 0.050 & 0.049 & 0.048 & 0.025 & $0 . \mathrm{C}$ \\
\hline SNP81 & $\begin{array}{l}\text { F: TTCTGTTAGACGAGATGCCG } \\
\text { R: AACTCTTGGCTCATGCGTAG } \\
\text { E: } \\
\text { gactgactgactgactgactgactgactgactgactgactCTGTTTAATATCGACTGATATTTC }\end{array}$ & 280 & $\mathrm{~T} / \mathrm{A}$ & 1.374 & 0.175 & 0.272 & 0.235 & 0.163 & $0 . C$ \\
\hline SNP82 & $\begin{array}{l}\text { F: CCAAATGTGATGGGTCTGTC } \\
\text { R: TGCTGCTTGACTCTCAGATG } \\
\text { E: ctgactgactgactTTAAGTAAGCCTGTCCAGAAAA }\end{array}$ & 274 & $\mathrm{~T} / \mathrm{A}$ & 1.632 & 0.275 & 0.387 & 0.312 & 0.263 & $0 . C$ \\
\hline SNP83 & $\begin{array}{l}\text { F: GCATTTTTGCAATATCCCCCA } \\
\text { R: GTCTGTGTGAGGGTGGCTTT } \\
\text { E: CACAAAATAATTGACCCTAGCTCCA }\end{array}$ & 486 & $\mathrm{~T} / \mathrm{C}$ & 1.977 & 0.107 & 0.494 & 0.3720 & 0.446 & $0 . C$ \\
\hline SNP84 & $\begin{array}{l}\text { F: TTATCAGACACCAAACATCCGAAGT } \\
\text { R: AAAGCACACTACTAGCCCACACA } \\
\text { E: ctTTCTAGATTCCGGGAGTGATTGTA }\end{array}$ & 227 & $T / G$ & 1.576 & 0.333 & 0.366 & 0.299 & 0.241 & 0.6 \\
\hline SNP85 & $\begin{array}{l}\text { F: TCTGTGCCCATCATATCGGC } \\
\text { R: CTGAAAGACCTGAGGCTGGG } \\
\text { E: ctgactgactgactAAAAGCCACAACGCCACCTCC }\end{array}$ & 254 & $\mathrm{~A} / \mathrm{G}$ & 1.308 & 0.182 & 0.236 & 0.2080 & 0.136 & 0.2 \\
\hline
\end{tabular}

Table 1 (continued) 


\begin{tabular}{|c|c|c|c|c|c|c|c|c|c|}
\hline $\begin{array}{l}\text { Prime } \\
\text { ID }\end{array}$ & Primer sequence $\left(5^{\prime}-3^{\prime}\right)$ & $\begin{array}{l}\text { Size } \\
\text { (bp) }\end{array}$ & $\begin{array}{l}\text { SNP } \\
\text { type }\end{array}$ & $\mathrm{Ne}$ & Ho & $\mathrm{He}$ & $P I C$ & MAF & $P_{\mathrm{HU}}$ \\
\hline SNP86 & $\begin{array}{l}\text { F: CGAGCGAGCGAATGTTTGTGAA } \\
\text { R: TGGTTTAGCCATTCTTCTGTGTTGG } \\
\text { E: actgactgactgactCTACGACTAGGCCTCССTCCCT }\end{array}$ & 315 & $A / G$ & 1.576 & 0.333 & 0.366 & 0.299 & 0.241 & 0.64 \\
\hline SNP87 & $\begin{array}{l}\text { F: CGGTGTGAAGAGCGTCCATA } \\
\text { R: CTCTTCTGCCTTGTCCACGT } \\
\text { E: tgactgactgactTTGTAGGGGGGTCTGTGTACAAGG }\end{array}$ & 393 & $\mathrm{~T} / \mathrm{A}$ & 1.800 & 0.296 & 0.444 & 0.3460 & 0.333 & $0.0 \varepsilon$ \\
\hline SNP88 & $\begin{array}{l}\text { F: TGGCTTTAAGAATGTCTGAGTGTGT } \\
\text { R: GCTCAATAGCATGACGGAGGTG } \\
\text { E: tgactgactgactgactgactAAACTATTTACTAGATCTCCTCT }\end{array}$ & 245 & $A / G$ & 1.906 & 0.556 & 0.475 & 0.362 & 0.389 & $0.3 \varepsilon$ \\
\hline SNP89 & $\begin{array}{l}\text { F: AGCCAATGCTGCCATTCCATACAA } \\
\text { R: GATCGGTTCTGTGCCAGGACAC } \\
\text { E: ctgactgactgactgactgactAAAAGTCTCCACGTATACTCCATCACT }\end{array}$ & 263 & $\mathrm{G} / \mathrm{A}$ & 1.957 & 0.333 & 0.489 & 0.3690 & 0.426 & $0.0 \subseteq$ \\
\hline SNP90 & $\begin{array}{l}\text { F: TGTGTGCTCTCTGTGTATGTGTTCA } \\
\text { R: TCAGTGCTGTTAGGTATTGTGGGTA } \\
\text { E: tgactgactgactgactgactgactGTGATTTTTGTTTCTTATTTCTTATAAC }\end{array}$ & 331 & $\mathrm{~A} / \mathrm{T}$ & 1.385 & 0.037 & 0.278 & 0.239 & 0.167 & $0.0 \mathrm{C}$ \\
\hline SNP91 & $\begin{array}{l}\text { F: TTAGGCTGGCTACATTGTCCATACG } \\
\text { R: AGCGGAGAGCGACTGTCACT } \\
\text { E: actgactgactgactgactgactgactgactgactAGAACACATCCTCTCCAGCATG }\end{array}$ & 217 & $\mathrm{~A} / \mathrm{T}$ & 1.839 & 0.259 & 0.456 & 0.3520 & 0.352 & 0.02 \\
\hline SNP92 & $\begin{array}{l}\text { F: TTGTCCGCAGTGCTAAGCAGTTA } \\
\text { R: CGCTTTACCAGTGTCCCGCTAA } \\
\text { E: } \\
\text { tgactgactgactgactgactgactgactgactCAGCATAGGTATGTTATTCTTAAACATC }\end{array}$ & 334 & $\mathrm{~T} / \mathrm{C}$ & 1.989 & 0.556 & 0.497 & 0.374 & 0.463 & 0.54 \\
\hline SNP93 & $\begin{array}{l}\text { F: GCAAGTGGTGGAGGTGAAAGTGT } \\
\text { R: CGGATGCTTCCCTGGTATGTTGAA } \\
\text { E: TATTTAAAGCTCTTACCTCCTCTCC }\end{array}$ & 284 & $\mathrm{~T} / \mathrm{C}$ & 1.839 & 0.333 & 0.456 & 0.3520 & 0.352 & $0.1 \epsilon$ \\
\hline SNP94 & $\begin{array}{l}\text { F: TGGCAGTGCTCAAACCTCAA } \\
\text { R: GAAGGATGGCCACAGTGTCA } \\
\text { E: actCAAACCTCACTTGATGACTTCGAATT }\end{array}$ & 314 & $T / G$ & 1.800 & 0.444 & 0.444 & 0.346 & 0.333 & $1.0 \mathrm{C}$ \\
\hline SNP95 & $\begin{array}{l}\text { F: ATCCACTCCGATGCTGATGTAAGAT } \\
\text { R: GTGAGACCAGGCAGAAATGAAAGC } \\
\text { E: ctgactgactGCGATGACTCAGCCTCCAAGTGT }\end{array}$ & 231 & $\mathrm{C} / \mathrm{A}$ & 1.246 & 0.074 & 0.198 & 0.1780 & 0.111 & $0.0 C$ \\
\hline SNP96 & $\begin{array}{l}\text { F: TCTCAGTTACACAAAGACGCACACA } \\
\text { R: TCAGTGAGCAAGGCATCTTAGACAA } \\
\text { E: tgactgactgactgactCTACGCAAACAAACACACACAC }\end{array}$ & 280 & $\mathrm{~A} / \mathrm{T}$ & 1.715 & 0.222 & 0.417 & 0.330 & 0.296 & 0.01 \\
\hline SNP97 & $\begin{array}{l}\text { F: GCCTTTCCTCTTCCCGCCTAATC } \\
\text { R: GATCACATCGCCGTGTACGGTAAT } \\
\text { E: actgactgactgactgactAGATCACAAAAACTCCCGCTAC }\end{array}$ & 294 & $\mathrm{~T} / \mathrm{C}$ & 1.759 & 0.259 & 0.431 & 0.3380 & 0.315 & $0.0 ₹$ \\
\hline SNP98 & $\begin{array}{l}\text { F: GCCGCCACTGAGCAATATTACG } \\
\text { R: TTGGACTGCAACACTGCATTCA } \\
\text { E: tgactgactgactgactgactTAAGACTTGTTTAATTTCCAAACC }\end{array}$ & 336 & $\mathrm{G} / \mathrm{A}$ & 1.989 & 0.185 & 0.497 & 0.374 & 0.463 & $0.0 \mathrm{C}$ \\
\hline SNP99 & $\begin{array}{l}\text { F: TCATTGGACCTTGCTCTTTCAAAGC } \\
\text { R: GTGTCACATCAAACATTCACGCTGA } \\
\text { E: gactgactgactgactgactgactgactAGTGTCCACTGGCTCTCCATT }\end{array}$ & 327 & $\mathrm{~T} / \mathrm{A}$ & 1.839 & 0.259 & 0.456 & 0.3520 & 0.352 & 0.02 \\
\hline SNP100 & $\begin{array}{l}\text { F: GACTCAGACCATCACTCCACCTACA } \\
\text { R: TCCACAGAACCTGTCACTCAATGC } \\
\text { E: actgactgactgactgactgactgactgactAGCAGGTGAACAACACAGCCAC }\end{array}$ & 312 & $\mathrm{~T} / \mathrm{A}$ & 1.576 & 0.407 & 0.366 & 0.299 & 0.241 & 0.55 \\
\hline
\end{tabular}

Table 1 (continued) 


\begin{tabular}{|c|c|c|c|c|c|c|c|c|c|}
\hline $\begin{array}{l}\text { Prime } \\
\text { ID }\end{array}$ & Primer sequence $\left(5^{\prime}-3^{\prime}\right)$ & $\begin{array}{l}\text { Size } \\
\text { (bp) }\end{array}$ & $\begin{array}{l}\text { SNP } \\
\text { type }\end{array}$ & $\mathrm{Ne}$ & Ho & $\mathrm{He}$ & $P I C$ & MAF & $P_{\mathrm{HW}}$ \\
\hline SNP101 & $\begin{array}{l}\text { F: CCGTTACTGCCCACCTTAGTTATTG } \\
\text { R: ATGTTTGACGCAGATGTTGTTGATC } \\
\text { E: tgactgactgactgactgactgactgactTGTTATGGAAAGGAGATGTTGTTCCTGT }\end{array}$ & 316 & $\mathrm{C} / \mathrm{A}$ & 1.874 & 0.667 & 0.466 & 0.358 & 0.370 & 0.02 \\
\hline SNP102 & $\begin{array}{l}\text { F: AGACATCTTTTTGGCACATGTG } \\
\text { R: TTATGTGCTGTGCTGGGACC } \\
\text { E: } \\
\text { gactgactgactgactgactgactgactgactgactgactTGGCACATGTGCAGCTTAAAC }\end{array}$ & 317 & $\mathrm{G} / \mathrm{C}$ & 1.957 & 0.259 & 0.489 & 0.3690 & 0.426 & 0.01 \\
\hline SNP103 & $\begin{array}{l}\text { F: TTCTGGACCAGCAGGACTTCATTG } \\
\text { R: GGTTGATTCTGACTGACAGGTGAGA } \\
\text { E: AAATGAAATTGGTCACGAATGCTCC }\end{array}$ & 289 & $\mathrm{C} / \mathrm{T}$ & 1.989 & 0.630 & 0.497 & 0.374 & 0.463 & 0.16 \\
\hline SNP104 & $\begin{array}{l}\text { F: GCAACCTGACAAGCCATACTTTCCA } \\
\text { R: ACCGTCCATCCTGATGTACTGTGTA } \\
\text { E: tAAGACTAAAACAACATACTTGTTCTCCT }\end{array}$ & 336 & $\mathrm{C} / \mathrm{T}$ & 1.843 & 0.125 & 0.457 & 0.3530 & 0.354 & 0.00 \\
\hline SNP105 & $\begin{array}{l}\text { F: AGCCCGGTCTGGTCGTATTTGA } \\
\text { R: AGCCCTTACTCGATGTGCATTTGAT } \\
\text { E: tgactCATTCAAATGTTGAAATATGAGACAGGC }\end{array}$ & 283 & $\mathrm{G} / \mathrm{T}$ & 2.000 & 0.333 & 0.500 & 0.375 & 0.500 & 0.08 \\
\hline SNP106 & $\begin{array}{l}\text { F: TGCTAGTGTGATAACTGCTTGTAGA } \\
\text { R: CCGTTGCTGGATATGGCTGTTG } \\
\text { E: actgactgactgactGCAGTCGGAAAGCTTGGACAGC }\end{array}$ & 286 & $\mathrm{~A} / \mathrm{T}$ & 1.839 & 0.556 & 0.456 & 0.3520 & 0.352 & 0.25 \\
\hline SNP107 & $\begin{array}{l}\text { F: TTGTGTAGCCAGTTTCTCAGCGAAT } \\
\text { R: AAGCATGAACATAAAGCAGGTGGGT } \\
\text { E: actgactgactgactAAAAGGTTTACTGTAACTCCATCCCA }\end{array}$ & 215 & $\mathrm{~T} / \mathrm{C}$ & 1.715 & 0.296 & 0.417 & 0.330 & 0.296 & 0.13 \\
\hline SNP108 & $\begin{array}{l}\text { F: AGCTGATTCCAGACAAATACATACG } \\
\text { R: GTGATAACAACCGGATGCCTATACA } \\
\text { E: ctgactgactgactgactAAAAGAAAATAGCTACTCACCCTCCCA }\end{array}$ & 334 & $\mathrm{G} / \mathrm{A}$ & 1.989 & 0.407 & 0.497 & 0.3740 & 0.481 & 0.34 \\
\hline SNP109 & $\begin{array}{l}\text { F: TGATATGGTACTCCTGCTTGGTAGG } \\
\text { R: GTAACGGGTCGTGACGTTTGTC } \\
\text { E: ctgactgactgactgactgactgactTCCACATCAATGGAGACGTGGTT }\end{array}$ & 292 & $\mathrm{G} / \mathrm{A}$ & 1.874 & 0.370 & 0.466 & 0.358 & 0.370 & 0.28 \\
\hline SNP110 & $\begin{array}{l}\text { F: TTATGGCTATGGAGGCTGTTGAATT } \\
\text { R: TTGGTATAGGTTGTTACTCTGTGGT } \\
\text { E: tgactgactgactgactgactgactgactTCACCTTAGCAAAAAGAGCTCGGC }\end{array}$ & 350 & $\mathrm{~A} / \mathrm{G}$ & 1.906 & 0.407 & 0.475 & 0.3620 & 0.389 & 0.45 \\
\hline SNP111 & $\begin{array}{l}\text { F: AGCTGGGTTAGACTTCTAAGCACAT } \\
\text { R: TTATCGAGCGAAGTTTACGTTTGGT } \\
\text { E: ctgactgactgactgactgactgactgactgactCAGCTGCCTGCGATGAAAACACA }\end{array}$ & 334 & $\mathrm{~A} / \mathrm{T}$ & 1.874 & 0.444 & 0.466 & 0.358 & 0.370 & 0.80 \\
\hline SNP112 & $\begin{array}{l}\text { F: ACATTGCATCATGGAACGGTGTTG } \\
\text { R: GGACTCACTCGGAGACCAGGAA } \\
\text { E: } \\
\text { gactgactgactgactgactgactgactgactgactgactCGGACGGAAATGAGGTACAGG }\end{array}$ & 311 & $\mathrm{G} / \mathrm{C}$ & 1.976 & 0.444 & 0.494 & 0.3720 & 0.444 & 0.60 \\
\hline
\end{tabular}

Sequences and additional SNP information can be found in Supplementary Material.

Primers for each locus including a forward primer (F), a reverse primer (R) and an extension primer (E), Ho observed heterozygosity, He expected heterozygosity, $P I C$ polymorphism information content, MAF minor allele frequency, $P_{\mathrm{HWE}} P$-values for Hardy-Weinberg equilibrium corrected for multiple comparisons using the false discovery rate.

* Signifcantly deviated from Hardy-Weinberg equilibrium $(P<0.05)$, ** Signifcantly deviated from Hardy-Weinberg equilibrium $(P<0.01)$, ** Signifcantly deviated from Hardy-Weinberg equilibrium $(P<0.001)$.

\section{Supplementary Files}

This is a list of supplementary files associated with this preprint. Click to download.

- Supplementaryinformation.docx 